\title{
Effects of Virulence Factors On Streptococcus Pneumoniae in Vitro Growth
}

Nor Munirah Mohd Amin ${ }^{1}$, Mohammad Izwan Enche Othman ${ }^{1}$, Engku Anis Fariha Engku Nasrullah Satiman ${ }^{1}$, Nur Jannah Abdul Aziz ${ }^{1}$, Aisyah Mohamed Rehan², Intan Azura Shahdan ${ }^{1}$, Nor Saadah Md. Azahri ${ }^{1}$, Mohd Nasir Mohd Desa ${ }^{3}$, Hanani Ahmad Yusof Hanafi ${ }^{1}$

${ }^{1}$ Department of Biomedical Science, Kulliyyah of Allied Health Sciences, International Islamic University Malaysia

${ }^{2}$ Department of Biotechnology, Kulliyyah of Science, International Islamic University Malaysia

${ }^{3}$ Department of Biomedical Science, Faculty of Medicine and Health Sciences, Universiti Putra Malaysia.

Presenter: Nor Munirah Mohd Amin

Introduction: Streptococcus pneumoniae remains as a major causative agent for morbidity and mortality cases in the world. The pathogenicity of this bacterium is attributed to many virulence factors. These factors, though not all had been extensively investigated at the molecular level in various mutant studies. However, of these studies, none of them were using local Malaysian isolate. Materials and Methods: Therefore, to better understand the growth pattern of local mutants a growth curve study and optochin disk susceptibility test were conducted. Several virulence genes (i.e hyl, ugl, $\operatorname{ccpA}$, nanA, and ply) were targeted for this local mutant construction. Pneumococcal mutants were constructed by employing genome-wide deletion technique using high throughput polymerase chain reaction (PCR). Linear knockout construct was established by fusing the kanamycin resistance protein encoding gene, aphA-3 with $1 \mathrm{~Kb}$ homologous sequence up and down stream of the respective targeted genes. The gene was knocked out by allelic replacement mechanism. Results: Glycosaminoglycan (GAG) gene cluster mutants ( $\Delta$ hyl- and $\Delta$ ugl-) took two days compared to 3 others to grow on brain heart infusion $(\mathrm{BHI})$-kanamycin agar plate following transformation. This indicated a variance in the mutant growth pattern. Therefore, a growth curve study was conducted to validate the previous observation. We report different time span of log phase in growth curve pattern of mutants relative to the wild type strain. Meanwhile, comparison of zone of inhibition using optochin disk showed non-identical inhibition diameter $(\geq 14 \mathrm{~mm})$ corresponding to the wild type $(14.3 \mathrm{~mm})$. Conclusion: The difference in growth pattern was attributed to nutritional role of certain gene. 